\title{
\$31. Protochips and parachips
}

Recall that for any prechip e we have put

$$
e[B]=e(B, B, 0) \text { for } 1 \leq B \leq o(\ell(e))
$$

and

$$
e[[B]]=\underset{\substack{l \leq b \leq 0(\ell(e)) \\ 0 \leq c \leq b(\ell(e))}}{\sum} e(B, b, c) \quad \text { for } \quad I \leq B \leq O(\ell(e)) .
$$

By a protochip we mean a prechip e such that

$$
e[B]] \neq 0 \text { for } 1 \leq B \leq o(\ell(e)) \text {. }
$$

By a parachip we mean a protochip e such that

$$
\text { e[B] } \neq 0 \text { for } 1 \leq B \leq 0(\ell(e))-2 \text {. }
$$

